\title{
Stability Analysis of Switched Time-Delay Systems
}

\author{
Peng Yan and Hitay Özbay
}

\begin{abstract}
This paper addresses the asymptotic stability of switched time delay systems with heterogenous time invariant time delays. Piecewise Lyapunov-Razumikhin functions are introduced for the switching candidate systems to investigate the stability in the presence of infinite number of switchings. We provide sufficient conditions in terms of the minimum dwell time to guarantee asymptotic stability under the assumptions that each switching candidate is delay-independently or delaydependently stable. Conservatism analysis is also provided by comparing with the dwell time conditions for switched delay free systems.
\end{abstract}

\section{INTRODUCTION}

Switching control offers a new look into the design of complex control systems (e.g. nonlinear systems, parameter varying systems and uncertain systems) [1], [8], [9], [19], [17], [21], [28]. Unlike the conventional adaptive control techniques that rely on continuous tuning, the switching control method updates the controller parameters in a discrete fashion based on the switching logic. The resulting closedloop systems have hybrid behaviors (e.g. continuous dynamics, discrete time dynamics and jump phenomena, etc.). One of the most challenging issues in the area of hybrid systems is the stability analysis in the presence of control switching. We refer to [9] for a general review on switching control methods.

In particular, we are interested in the stability analysis of switched time delay systems. In fact, time delay systems are ubiquitous in chemical processes, aerodynamics, and communication networks [3], [14]. To further complicate the situation, the time delays are usually time varying and uncertain [24], [25]. It has been shown that robust $\mathcal{H}^{\infty}$ controllers can be designed for such infinite dimensional plants, where robustness can be guaranteed within some uncertainty bounds [4]. In order to incorporate larger operating range or better robustness, controller switching can be introduced, which results in switched closed-loop systems with time delays. For delay free systems, stability analysis and design methodology have been investigated recently in the framework of hybrid dynamical systems [1], [2], [8], [11], [19], [21], [26]. In particular, [21] provided sufficient conditions on the stability of the switching control systems based on Filippov solutions to discontinuous differential equations and Lyapunov functionals; [19] proposed a dwell-time based switching control, where a sufficiently large dwell-time can

This work is supported in part by by TÜBİTAK under grant no. EEEAG$105 \mathrm{E} 156$.

P. Yan is with Enterprise Design Center, Seagate Technology LLC, 1280 Disc Drive, Shakopee, MN 55379, USA Peng.Yan@seagate.com

H. Özbay is with Dept. of Electrical \& Electronics Engineering, Bilkent University, Ankara 06800, Turkey hitay@bilkent.edu.tr guarantee the system stability. A more flexible result was obtained in [10], where the average dwell-time was introduced for switching control. In [26] the results of [10] were extended to LPV systems. LaSalle's invariance principle was extended to a class of switched linear systems for stability analysis [8]. Despite the variety and significance of the many results on hybrid system stability, stability of switched time delay systems hasn't been adequately addressed due to the general difficulty of infinite dimensional systems [7].

Two important approaches in the stability analysis of time delay systems are (1) Lyapunov-Krasovskii method, and (2) Lyapunov-Razumikhin method [6], [20]. Various sufficient conditions with respect to the stability of time delay systems have been given using Riccati-type inequalities or LMIs [3], [12], [14], [24]. In the meanwhile, stability analysis in the presence of switching has been discussed in some recent works [16], [18], [22]. In [18] stability and stabilizability were discussed for discrete time switched time delay systems; [16] considered similar stability problem in continuous time domain. Note that [18] and [16] are trajectory dependent results without taking admissible switching signals into considerations.

The main contribution of this paper is a collection of results on the trajectory independent stability of continuous time switched time delay systems using piecewise LyapunovRazumikhin functions. The dwell time of the switching signals is constructively given, which guarantees asymptotic stability for the delay independent case and the delay dependent case, respectively. Note that the asymptotic stability of finite dimensional linear systems indicates exponential stability, whereas this is not the case for infinite dimensional systems, [7], [15]. This poses the key challenge in the analysis of switched time delay systems.

The paper is organized as follows. The problem is defined in Section II. In Section III, the main results on the stability of switched time delay systems are presented in terms of the dwell time of the switching signals. Conservatism analysis is provided by comparing with the dwell time conditions for switching delay free systems in Section IV, followed by concluding remarks in Section V.

\section{Problem Definition}

For convenience, we would like to employ the following notation. The general Retarded Functional Differential Equations (RFDE) with time delay $r$ can be described as

$$
\dot{x}(t)=f\left(t, x_{t}\right)
$$

with initial condition $\phi(\cdot) \in C\left([-r, 0], \mathbb{R}^{n}\right)$, where $x_{t}$ denotes the state defined by $x_{t}(\theta)=x(t+\theta),-r \leq \theta \leq 0$. 
We use $\|\cdot\|$ to denote the Euclidean norm of a vector in $\mathbb{R}^{n}$, and $|f|_{[t-r, t]}$ for the $\infty$-norm of $f$, i.e.

$$
|f|_{[t-r, t]}:=\sup _{t-r \leq \theta \leq t}\|f(\theta)\|,
$$

where $f$ is an element of the Banach space $C\left([t-r, t], \mathbb{R}^{n}\right)$.

Consider the following switched time delay systems:

$\Sigma_{t}:\left\{\begin{array}{c}\dot{x}(t)=A_{q(t)} x(t)+\bar{A}_{q(t)} x\left(t-\tau_{q(t)}\right), \quad t \geq 0 \\ x_{0}(\theta)=\phi(\theta), \quad \forall \theta \in\left[-\tau_{\max }, 0\right]\end{array}\right.$

where $x(t) \in \mathbb{R}^{n}$ and $q(t)$ is a piecewise switching signal taking values on the set $\mathcal{F}:=\{1,2, \ldots, l\}$, i.e. $q(t)=k_{j}$, $k_{j} \in \mathcal{F}$, for $\forall t \in\left[t_{j}, t_{j+1}\right)$, where $t_{j}, j \in \mathbb{Z}^{+} \cup\{0\}$, is the $j^{\text {th }}$ switching time instant. It is clear that the trajectory of $\Sigma_{t}$ in any arbitrary switching interval $t \in\left[t_{j}, t_{j+1}\right)$ obeys:

$\Sigma_{k_{j}}:\left\{\begin{array}{c}\dot{x}(t)=A_{k_{j}} x(t)+\bar{A}_{k_{j}} x\left(t-\tau_{k_{j}}\right), t \in\left[t_{j}, t_{j+1}\right) \\ x_{t_{j}}(\theta)=\phi_{j}(\theta), \quad \forall \theta \in\left[-\tau_{k_{j}}, 0\right],\end{array}\right.$

where $\phi_{j}(\theta)$ is defined as:

$$
\phi_{j}(\theta)=\left\{\begin{array}{lr}
x\left(t_{j}+\theta\right) & -\tau_{k_{j}} \leq \theta<0 \\
\lim _{h \rightarrow 0^{-}} x\left(t_{j}+h\right), & \theta=0
\end{array}\right.
$$

We introduce the triplet $\Sigma_{i}:=\left(A_{i}, \bar{A}_{i}, \tau_{i}\right) \in \mathbb{R}^{n \times n} \times$ $\mathbb{R}^{n \times n} \times \mathbb{R}^{+}$to describe the $i^{t h}$ candidate system of (2). Thus for $\forall t \geq 0$, we have $\Sigma_{t} \in \mathcal{A}:=\left\{\Sigma_{i}: i \in \mathcal{F}\right\}$, where $\mathcal{A}$ is the family of candidate systems of (2). In (2), $\phi(\cdot):\left[-\tau_{\max }, 0\right] \rightarrow \mathbb{R}^{n}$ is a continuous and bounded vectorvalued function, where $\tau_{\max }=\max _{i \in \mathcal{F}}\left\{\tau_{i}\right\}$ is the maximal time delay of the candidate systems in $\mathcal{A}$.

Similar to [8], we say that the switched time-delay system $\Sigma_{t}$ described by (2) is stable if there exists a function $\bar{\alpha}$ of class $\mathcal{K}^{1}$ such that

$$
\|x(t)\| \leq \bar{\alpha}\left(|x|_{\left[t_{0}-\tau_{\text {max }}, t_{0}\right]}\right), \quad \forall t \geq t_{0} \geq 0,
$$

along the trajectory of (2). Furthermore, $\Sigma_{t}$ is asymptotically stable when $\Sigma_{t}$ is stable and $\lim _{t \rightarrow+\infty} x(t)=0$.

Lemma 2.1: ([3], [14]) Suppose for a given triplet $\Sigma_{i} \in$ $\mathcal{A}, i \in \mathcal{F}$, there exists symmetric and positive-definite $P_{i} \in$ $\mathbb{R}^{n \times n}$, such that the following LMI with respect to $P_{i}$ is satisfied for some $p_{i}>1$ and $\alpha_{i}>0$ :

$$
\left[\begin{array}{cc}
P_{i} A_{i}+A_{i}^{T} P_{i}+p_{i} \alpha_{i} P_{i} & P_{i} \bar{A}_{i} \\
\bar{A}_{i}^{T} P_{i} & -\alpha_{i} P_{i}
\end{array}\right]<0 .
$$

Then $\Sigma_{i}$ is asymptotically stable independent of delay.

If all candidate systems of (2), $\Sigma_{i} \in \mathcal{A}$, are delayindependently asymptotically stable satisfying (6), we denote $\mathcal{A}$ by $\tilde{\mathcal{A}}$.

Lemma 2.2: ([3], [14]) Suppose for a given triplet $\Sigma_{i} \in$ $\mathcal{A}, i \in \mathcal{F}$, there exists symmetric and positive-definite $P_{i} \in$ $\mathbb{R}^{n \times n}$, and a scalar $p_{i}>1$, such that

$$
\left[\begin{array}{cc}
\tau_{i}^{-1} \Omega_{i} & P_{i} \bar{A}_{i} M_{i} \\
M_{i}^{T} \bar{A}_{i}^{T} P_{i} & -R_{i}
\end{array}\right]<0
$$

\footnotetext{
${ }^{1}$ A continuous function $\bar{\alpha}(\cdot): \mathbb{R}^{+} \rightarrow \mathbb{R}^{+}$is a class $\mathcal{K}$ function if it is strictly increasing and $\bar{\alpha}(0)=0$.
}

where

$$
\begin{aligned}
\Omega_{i} & =\left(A_{i}+\bar{A}_{i}\right)^{T} P_{i}+P_{i}\left(A_{i}+\bar{A}_{i}\right)+\tau_{i} p_{i}\left(\alpha_{i}+\beta_{i}\right) P_{i}, \\
M_{i} & =\left[A_{i} \bar{A}_{i}\right], \\
R_{i} & =\operatorname{diag}\left(\alpha_{i} P_{i}, \beta_{i} P_{i}\right),
\end{aligned}
$$

and $\alpha_{i}>0, \beta_{i}>0$ are scalars. Then $\Sigma_{i}$ is asymptotically stable dependent of delay.

Similarly we denote $\mathcal{A}$ by $\tilde{\mathcal{A}}_{d}$ if all candidate systems of (2) are delay-dependently asymptotically stable satisfying (7).

In what follows, we will establish sufficient conditions to guarantee stability of switched system (2) for the delay independent case and the delay dependent case. Therefore, we will assume that $\mathcal{A}=\tilde{\mathcal{A}}$ and $\mathcal{A}=\tilde{\mathcal{A}}_{d}$ respectively in the corresponding sections in this paper. An important method in stability analysis of switched systems is based on the construction of the common Lyapunov function (CLF), which allows for arbitrary switching. However, this method is too conservative from the perspective of controller design because it is usually difficult to find the CLF for all the candidate systems, particularly for time delay systems whose stability criteria are only sufficient in most of the circumstances. A recent paper [29] explored the CLF method for switched time delays systems with three very strong assumptions: (i) each candidate system has the same time delay $\tau$; (ii) each candidate is assumed to be delay independently stable; (iii) The $A$-matrix is always symmetric and the $\bar{A}$ matrix is always in the form of $\delta I$. In the present paper, we consider an alternative method using piecewise LyapunovRazumikhin functions for a general class of systems (2) and obtain stability conditions in terms of the dwell time of the switching signal. This method can be used for the case with delay independent criterion (6) and the case with delay dependent criterion (7).

\section{Main Results on Dwell Time Based Switching}

For a given positive constant $\tau_{D}$, the switching signal set based on the dwell time $\tau_{D}$ is denoted by $S\left[\tau_{D}\right]$, where for any switching signal $q(t) \in S\left[\tau_{D}\right]$, the distance between any consecutive discontinuities of $q(t), t_{j+1}-t_{j}, j \in \mathbb{Z}^{+} \cup$ $\{0\}$, is larger than $\tau_{D}$ [10], [19]. Sufficient condition on the minimum dwell time to guarantee the stable switching will be given using piecewise Lyapunov-Razumikhin functions. Note that the dwell time based switching is trajectory-independent [8].

Before presenting the main result of this paper, we recall the following lemma [7] for general Retarded Functional Differential Equations (1).

Lemma 3.1: [7] Suppose $u, v, w, p: \mathbb{R}^{+} \rightarrow \mathbb{R}^{+}$are continuous,nondecreasing functions, $u(0)=v(0)=0$, $u(s), v(s), w(s), p(s)$ positive for $s>0, p(s)>s$, and $v(s)$ strictly increasing. If there is a continuous function $V: \mathbb{R} \times \mathbb{R}^{n} \rightarrow \mathbb{R}$ such that

$$
u(\|x(t)\|) \leq V(t, x) \leq v(\|x(t)\|), \quad t \in \mathbb{R}, x \in \mathbb{R}^{n},
$$

and

$$
\dot{V}(t, x(t)) \leq-w(\|x(t)\|),
$$


if

$$
V(t+\theta, x(t+\theta))<p(V(t, x(t))) \quad \forall \theta \in[-r, 0],
$$

then the solution $x=0$ of the RFDE is uniformly asymptotically stable.

A particular case of (1) is a linear time delay system $\Sigma_{i}, i \in \mathcal{F}$, where we can construct the corresponding Lyapunov-Razumikhin function in the quadratic form

$$
V_{i}(t, x)=x^{T}(t) P_{i} x(t), \quad P_{i}=P_{i}^{T}>0 .
$$

Apparently $V_{i}$ can be bounded by

$$
u_{i}(\|x(t)\|) \leq V_{i}(t, x) \leq v_{i}(\|x(t)\|), \quad \forall x \in \mathbb{R}^{n},
$$

where

$$
u_{i}(s):=\kappa_{i} s^{2}, \quad v_{i}(s):=\bar{\kappa}_{i} s^{2}
$$

in which $\kappa_{i}:=\sigma_{\min }\left[P_{i}\right]>0$ denotes the smallest singular value of $P_{i}$ and $\bar{\kappa}_{i}:=\sigma_{\max }\left[P_{i}\right]>0$ the largest singular value of $P_{i}$.

Proposition 3.2: For each time delay systems $\Sigma_{i}$ with Lyapunov-Razumikhin function defined by (11) assume (9) and (10) are satisfied for some $w_{i}(s)$. Then we have

$$
|x|_{\left[t_{m}-\tau_{i}, t_{m}\right]} \leq\left(\frac{\bar{\kappa}_{i}}{\kappa_{i}}\right)^{1 / 2}|x|_{\left[t_{n}-\tau_{i}, t_{n}\right]}, \forall t_{m} \geq t_{n} \geq 0 .
$$

Proof. Define

$$
\bar{V}_{i}(t, x):=\sup _{-\tau_{i} \leq \theta \leq 0} V_{i}(t+\theta, x(t+\theta))
$$

for $t \geq 0$, we have

$$
\kappa_{i}\left(|x|_{\left[t-\tau_{i}, t\right]}\right)^{2} \leq \bar{V}_{i}(t, x) \leq \bar{\kappa}_{i}\left(|x|_{\left[t-\tau_{i}, t\right]}\right)^{2}, \quad t \geq 0
$$

The definition of $\bar{V}_{i}(t, x)$ implies $\exists \theta_{0} \in\left[-\tau_{i}, 0\right]$, such that $\bar{V}_{i}(t, x)=V\left(t+\theta_{0}, x\left(t+\theta_{0}\right)\right)$. Introduce the upper righthand derivative of $\bar{V}_{i}(t, x)$ as

$$
\dot{\bar{V}}_{i}^{+}=\limsup _{h \rightarrow 0^{+}} \frac{1}{h}\left[\bar{V}_{i}(t+h, x(t+h))-\bar{V}_{i}(t, x(t))\right],
$$

we have

(i). If $\theta_{0}=0$, i.e. $V_{i}(t+\theta, x(t+\theta)) \leq V_{i}(t, x(t))<$ $p\left(V_{i}(t, x(t))\right)$, we have $\dot{V}_{i}(t, x)<0$ by (9). Therefore $\dot{\bar{V}}_{i}^{+} \leq 0$.

(ii). If $-\tau_{i}<\theta_{0}<0$, we have $\bar{V}_{i}(t+h, x(t+h))=\bar{V}_{i}(t, x)$ for $h>0$ sufficiently small, which results in $\dot{\bar{V}}_{i}^{+}=0$.

(iii). If $\theta_{0}=-\tau_{i}$, the continuity of $V_{i}(t, x)$ implies $\dot{\bar{V}}_{i}^{+} \leq 0$.

The above analysis shows that

$$
\bar{V}_{i}\left(t_{m}\right) \leq \bar{V}_{i}\left(t_{n}\right), \quad \forall t_{m} \geq t_{n} \geq 0 .
$$

Recall (16), we have

$$
\kappa_{i}\left(|x|_{\left[t_{m}-\tau_{i}, t_{m}\right]}\right)^{2} \leq \bar{V}_{i}\left(t_{m}\right) \leq \bar{V}_{i}\left(t_{n}\right) \leq \bar{\kappa}_{i}\left(|x|_{\left[t_{n}-\tau_{i}, t_{n}\right]}\right)^{2},
$$

for any $t_{m} \geq t_{n} \geq 0$. This implies (14) and proves the result.

Suppose all of the conditions of Lemma 3.1 are satisfied for general RFDE (1), we also have the following result.
Lemma 3.3: [7] Suppose $|\phi|_{\left[t_{0}-r, t_{0}\right]} \leq \bar{\delta}_{1}, \bar{\delta}_{1}>0$, and $\bar{\delta}_{2}>0$ such that $v\left(\bar{\delta}_{1}\right)=u\left(\bar{\delta}_{2}\right)$. For all $\eta$ satisfying $0<$ $\eta \leq \bar{\delta}_{2}$, we have

$$
V(t, x) \leq u(\eta), \quad \forall t \geq t_{0}+T .
$$

Here

$$
T=\frac{N v\left(\bar{\delta}_{1}\right)}{\gamma}
$$

is defined by $\gamma=\inf _{v^{-1}(u(\eta)) \leq s \leq \bar{\delta}_{2}} w(s)$ and $N=\left\lceil\left(v\left(\bar{\delta}_{1}\right)-\right.\right.$ $u(\eta)) / a\rceil$, where $\lceil\cdot\rceil$ is the ceiling integer function and $a>0$ satisfies $p(s)-s>a$ for $u(\eta) \leq s \leq v\left(\bar{\delta}_{1}\right)$.

\section{A. The Case with Delay Independent Criterion}

Consider the switched time delay systems $\Sigma_{t}$ defined by (2) and assume each candidate system $\Sigma_{i}, i \in \mathcal{F}$ delay-independently asymptotically stable satisfying (6) (i.e. $\mathcal{A}=\tilde{\mathcal{A}})$. A sufficient condition on the minimum dwell time to guarantee the asymptotic stability can be derived using multiple piecewise Lyapunov-Razumikhin functions. In order to state the main result we make some preliminary definitions.

For the switched delay systems (2), first assume $\tau_{D}>$ $\tau_{\max }$. Consider an arbitrary switching interval $\left[t_{j}, t_{j+1}\right)$ of the piecewise switching signal $q(t) \in S\left[\tau_{D}\right]$, where $q(t)=$ $k_{j}, k_{j} \in \mathcal{F}$ for $\forall t \in\left[t_{j}, t_{j+1}\right)$ and $t_{j}$ is the $j^{t h}$ switching time instant for $j \in \mathbb{Z}^{+} \cup\{0\}$ and $t_{0}=0$. The state variable $x_{j}(t)$ defined on this interval obeys (3). For the convenience of using "sup", we define $x_{j}\left(t_{j+1}\right)=\lim _{h \rightarrow 0^{-}} x_{j}\left(t_{j+1}+h\right)=$ $x_{j+1}\left(t_{j+1}\right)$ based on the fact that $x(t)$ is continuous for $t \geq 0$. Therefore $x_{j}(t)$ is now defined on a compact set $\left[t_{j}, t_{j+1}\right]$. Recall (4), the initial condition $\phi_{j}(t)$ of $\Sigma_{k_{j}}$ is $\phi_{j}(t)=x(t)=x_{j-1}(t), t \in\left[t_{j}-\tau_{k_{j}}, t_{j}\right]$ for $j \in \mathbb{Z}^{+}$, which is true because $\tau_{D}>\tau_{\max }$.

Construct the Lyapunov-Razumikhin function

$$
V_{k_{j}}\left(x_{j}, t\right)=x_{j}^{T}(t) P_{k_{j}} x_{j}(t), \quad t \in\left[t_{j}, t_{j+1}\right]
$$

for (3), then we have

$$
\kappa_{k_{j}}\left\|x_{j}(t)\right\|^{2} \leq V_{k_{j}}\left(t, x_{j}\right) \leq \bar{\kappa}_{k_{j}}\left\|x_{j}(t)\right\|^{2}, \forall x_{j} \in \mathbb{R}^{n} .
$$

A straightforward calculation gives the time derivative of $V_{k_{j}}\left(t, x_{j}(t)\right)$ along the trajectory of (3)

$$
\begin{aligned}
\dot{V}_{k_{j}}\left(t, x_{j}\right)= & x_{j}^{T}\left(A_{k_{j}}^{T} P_{k_{j}}+P_{k_{j}} A_{k_{j}}\right) x_{j} \\
& +2 x_{j}^{T}(t) P_{k_{j}} \bar{A}_{k_{j}} x_{j}\left(t-\tau_{k_{j}}\right),
\end{aligned}
$$

where

$$
\begin{aligned}
& 2 x_{j}^{T}(t) P_{k_{j}} \bar{A}_{k_{j}} x_{j}\left(t-\tau_{k_{j}}\right) \\
\leq \quad & \alpha_{k_{j}} x_{j}^{T}\left(t-\tau_{k_{j}}\right) P_{k_{j}} x_{j}\left(t-\tau_{k_{j}}\right) \\
& +\alpha_{k_{j}}^{-1} x_{j}^{T}(t) P_{k_{j}} \bar{A}_{k_{j}} P_{k_{j}}^{-1} \bar{A}_{k_{j}}^{T} P_{k_{j}} x_{j}(t), \quad \forall \alpha_{k_{j}}>0 .
\end{aligned}
$$

Applying Razumikhin condition with $p(s)=p_{k_{j}} s, p_{k_{j}}>1$, we obtain

$$
x_{j}^{T}\left(t-\tau_{k_{j}}\right) P_{k_{j}} x_{j}\left(t-\tau_{k_{j}}\right) \leq p_{k_{j}} x_{j}^{T}(t) P_{k_{j}} x_{j}(t)
$$

for

$$
V_{k_{j}}\left(t+\theta, x_{j}(t+\theta)\right)<p_{k_{j}} V_{k_{j}}\left(t, x_{j}(t)\right) \quad \forall \theta \in\left[-\tau_{k_{j}}, 0\right] .
$$


Let

$$
\begin{aligned}
S_{k_{j}}:= & -\left(A_{k_{j}}^{T} P_{k_{j}}+P_{k_{j}} A_{k_{j}}+p_{k_{j}} \alpha_{k_{j}} P_{k_{j}}\right. \\
& \left.+\alpha_{k_{j}}^{-1} P_{k_{j}} \bar{A}_{k_{j}} P_{k_{j}}^{-1} \bar{A}_{k_{j}}^{T} P_{k_{j}}\right)
\end{aligned}
$$

we have

$$
\dot{V}_{k_{j}}\left(t, x_{j}\right) \leq-x_{j}^{T}(t) S_{k_{j}} x_{j}(t) .
$$

Because $\Sigma_{t} \in \tilde{\mathcal{A}}$, we have $S_{k_{j}}>0$ from Lemma 2.1. Furthermore we can select $w(s)=w_{k_{j}} s^{2}$ in Lemma 3.1, such that (9) is satisfied, where $w_{k_{j}}:=\sigma_{\min }\left[S_{k_{j}}\right]>0$.

Define

$$
\lambda:=\max _{i \in \mathcal{F}} \frac{\bar{\kappa}_{i}}{\kappa_{i}}
$$

and

$$
\mu:=\max _{i \in \mathcal{F}} \frac{\bar{\kappa}_{i}}{w_{i}} .
$$

Now we are ready to state the main result.

Theorem 3.4: Let the dwell time be defined by $\tau_{D}:=$ $T^{*}+\tau_{\max }$, where

$$
T^{*}:=\lambda \mu\left\lfloor\frac{\lambda-1}{\bar{p}-1}+1\right\rfloor,
$$

with $\bar{p}:=\min _{i \in \mathcal{F}}\left\{p_{i}\right\}>1$, and $\lfloor\cdot\rfloor$ being the floor integer function. Then the system (2) with $\Sigma_{t} \in \tilde{\mathcal{A}}$ is asymptotically stable for any switching rule $q(t) \in S\left[\tau_{D}\right]$.

Proof. First we claim that for all $\tau>\tau_{D}$, there exist $0<\beta<1$ and $0<\alpha<1$, such that $\tau \geq \bar{T}+\tau_{\max }$, where

$$
\bar{T}:=\frac{\lambda \mu}{\alpha^{2}}\left\lceil\frac{\lambda-\alpha^{2}}{\alpha^{2} \beta(\bar{p}-1)}\right\rceil .
$$

For a given $\tau$, to find such $\alpha$ and $\beta$ define $\tilde{T}+\tau_{\max }:=\tau>$ $\tau_{D}=T^{*}+\tau_{\max }$, and consider two cases below.

1) If $\lfloor(\lambda-1) /(\bar{p}-1)\rfloor=: k<(\lambda-1) /(\bar{p}-1)<k+1$, then can find $\Delta_{1}>0$ and $\Delta_{2}>0$ small enough, such that

$$
\left\lceil\frac{\lambda-\alpha_{1}^{2}}{\alpha_{1}^{2} \beta(\bar{p}-1)}\right\rceil=\left\lceil\frac{\lambda-1}{\bar{p}-1}\right\rceil=k+1=\left\lfloor\frac{\lambda-1}{\bar{p}-1}+1\right\rfloor
$$

with $\alpha_{1}=\left(1+\Delta_{1}\right)^{-\frac{1}{2}}<1$ and $\beta=\left(1+\Delta_{2}\right)^{-\frac{1}{2}}<1$. Let $\tilde{T}=T^{*}+\epsilon, \epsilon>0$. It is easy to check that

$\frac{\lambda \mu}{\alpha_{2}^{2}}\left\lceil\frac{\lambda-\alpha_{1}^{2}}{\alpha_{1}^{2} \beta(\bar{p}-1)}\right\rceil=\frac{\lambda \mu}{\alpha_{2}^{2}}(k+1) \leq(k+1) \lambda \mu+\epsilon=\tilde{T}$,

where $0<\alpha_{2}=\left(1+\Delta_{3}\right)^{-\frac{1}{2}}<1$ with $0<\Delta_{3} \leq$ $\frac{\epsilon}{(k+1) \lambda \mu}$. Now choosing $0<\alpha=\max \left\{\alpha_{1}, \alpha_{2}\right\}<1$, we have $\bar{T} \leq \tilde{T}$, which is straightforward from (30) and (31).

2) If $(\lambda-1) /(\bar{p}-1)=k>0$ is an integer. We can similarly find $0<\alpha_{1}<1$ and $0<\beta<1$ such that

$$
\left\lceil\frac{\lambda-\alpha_{1}^{2}}{\alpha_{1}^{2} \beta(\bar{p}-1)}\right\rceil=\left\lceil\frac{\lambda-1}{\bar{p}-1}+1\right\rceil=k+1=\left\lfloor\frac{\lambda-1}{\bar{p}-1}+1\right\rfloor
$$

In the same fashion as 1 ), we can constructively have $0<\alpha<1$ and $0<\beta<1$ such that $\bar{T} \leq \tilde{T}$.

This proves the first claim.

The second claim we make is that $\left\|x_{j}(t)\right\| \leq \alpha \delta_{j}$ for any $t \geq t_{j}+\bar{T}, t \in\left[t_{j}, t_{j+1}\right]$, where we assume $\left|\phi_{j}(t)\right|_{\left[t_{j}-\tau_{k_{j}}, t_{j}\right]} \leq \delta_{j}$. To show this fact, we can choose $\bar{\delta}_{1}=\delta_{j}, \bar{\delta}_{2}=\bar{\delta}_{1} \sqrt{\bar{\kappa}_{k_{j}} / \kappa_{k_{j}}} \geq \bar{\delta}_{1}$, and select $\eta=\alpha \bar{\delta}_{1}$ in Lemma 3.3. It is straightforward that $0<\eta<\bar{\delta}_{1} \leq \bar{\delta}_{2}$. Recall (19) and (20), we have

$$
V_{k_{j}}\left(t, x_{j}\right) \leq \kappa_{k_{j}} \eta^{2}, \text { for } t \geq t_{j}+T,
$$

where

$$
\begin{aligned}
T & =\frac{N v\left(\bar{\delta}_{1}\right)}{\gamma} \frac{\left\lceil\left(v\left(\bar{\delta}_{1}\right)-u(\eta)\right) / a\right\rceil v\left(\bar{\delta}_{1}\right)}{\inf _{v^{-1}(u(\eta)) \leq s \leq \bar{\delta}_{2}} w(s)} \\
& =\frac{\bar{\kappa}_{k_{j}}^{2}\left\lceil\left(v\left(\bar{\delta}_{1}\right)-u(\eta)\right) / a\right\rceil}{\alpha^{2} w_{k_{j}} \kappa_{k_{j}}}
\end{aligned}
$$

Combining (22) and (32) yields

$$
\left\|x_{j}(t)\right\| \leq \alpha \delta_{j}, \text { for } t \geq t_{j}+T .
$$

Now choosing $a=\beta\left(p_{k_{j}}-1\right) \kappa_{k_{j}} \eta^{2}$, we have

$$
T=\frac{\bar{\kappa}_{k_{j}}^{2}\left\lceil\frac{\frac{\bar{\kappa}_{k_{j}}}{\kappa_{k_{j}}}-\alpha^{2}}{\alpha^{2} \beta\left(p_{k_{j}}-1\right)}\right\rceil}{\alpha^{2} w_{k_{j}} \kappa_{k_{j}}} \leq \bar{T}
$$

Therefore from (34) and (35) we have

$$
\left|x_{j}\right|_{\left[t_{j}+\bar{T}, t_{j+1}\right]} \leq \alpha \delta_{j},
$$

as claimed.

Now recall that $t_{j+1}-t_{j}>\tau_{D}$. Therefore $t_{j+1}-t_{j} \geq$ $\bar{T}+\tau_{\max } \geq \bar{T}+\tau_{k_{j+1}}$. Also notice that $\phi_{j+1}(t)=x_{j}(t), t \in$ $\left[t_{j+1}-\tau_{k_{j+1}}, t_{j+1}\right]$. We have

$$
\begin{aligned}
& \left|\phi_{j+1}\right|_{\left[t_{j+1}-\tau_{k_{j+1}}, t_{j+1}\right]}=\left|x_{j}\right|_{\left[t_{j+1}-\tau_{k_{j+1}}, t_{j+1}\right]} \\
\leq & \left|x_{j}\right|_{\left[t_{j}+\bar{T}, t_{j+1}\right]} \leq \alpha \delta_{j}:=\delta_{j+1}
\end{aligned}
$$

and $\delta_{0}$ is defined as $\delta_{0}:=|\phi|_{\left[-\tau_{\max }, 0\right]} \geq|\phi|_{\left[-\tau_{k_{0}}, 0\right]}$. Therefore we obtain a convergent sequence $\left\{\delta_{i}\right\}, i=0,1,2, \ldots$, where $\delta_{i}=\alpha^{i} \delta_{0}$.

Meanwhile, (14) implies

$$
\left|x_{j}\right|_{\left[t-\tau_{k_{j}}, t\right]} \leq \sqrt{\frac{\bar{\kappa}_{k_{j}}}{\kappa_{k_{j}}}}\left|x_{j}\right|_{\left[t_{j}-\tau_{k_{j}}, t_{j}\right]}, \quad \forall t \in\left[t_{j}, t_{j+1}\right] .
$$

Hence

$$
\begin{aligned}
& \sup _{t \in\left[t_{j}, t_{j+1}\right]}\left\|x_{j}(t)\right\| \\
\leq & \sup _{t \in\left[t_{j}, t_{j+1}\right]}\left|x_{j}\right|_{\left[t-\tau_{k_{j}}, t\right]} \leq \sqrt{\lambda}\left|x_{j}\right|_{\left[t_{j}-\tau_{k_{j}}, t_{j}\right]} \\
\leq & \sqrt{\lambda} \delta_{j}=\alpha^{j} \sqrt{\lambda} \delta_{0},
\end{aligned}
$$

which implies the asymptotic stability of the switched time delay system $\Sigma_{t}$ with the switching signal $q(t) \in S_{\left[\tau_{D}\right]}$.

\section{B. The Case with Delay Dependent Criterion}

In a similar fashion, we can investigate the stability of the switched time delay system $\Sigma_{t}$ of (2) under the assumption that $\Sigma_{t} \in \overline{\mathcal{A}}_{d}$. Hence each candidate system $\Sigma_{i}, i \in \mathcal{F}$ is delay-dependently asymptotically stable satisfying (7). We assume $\tau_{D}^{d}>2 \tau_{\max }$ in this scenario. Similar to the proof of Theorem 3.4, we consider an arbitrary switching interval $\left[t_{j}, t_{j+1}\right)$ of the piecewise switching signal $q(t) \in S\left[\tau_{D}^{d}\right]$, 
where the state variable $x_{j}(t)$ defined on this interval obeys (3). The first order model transformation [7] of (3) results in

$$
\begin{aligned}
\dot{x}_{j}(t) & =\left(A_{k_{j}}+\bar{A}_{k_{j}}\right) x_{j}(t) \\
& -\bar{A}_{k_{j}} \int_{-\tau_{k_{j}}}^{0}\left[A_{k_{j}} x_{j}(t+\theta)+\bar{A}_{k_{j}} x\left(t+\theta-\tau_{k_{j}}\right)\right] d \theta
\end{aligned}
$$

where the initial condition $\psi_{j}(t)$ is defined as $\psi_{j}(t)=$ $x_{j-1}(t), t \in\left[t_{j}-2 \tau_{k_{j}}, t_{j}\right]$ for $j \in \mathbb{Z}^{+}$, and $\psi_{0}(t)$ defined by

$$
\psi_{0}(t)= \begin{cases}\phi(t), & t \in\left[-\tau_{\max }, 0\right] \\ \phi\left(-\tau_{\max }\right), & t \in\left[-2 \tau_{\max },-\tau_{\max }\right)\end{cases}
$$

By using the Lyapunov-Razumikhin function (21), we obtain the time derivative of $V_{k_{j}}\left(t, x_{j}(t)\right)$ along the trajectory of (40)

$$
\begin{aligned}
\dot{V}_{k_{j}}\left(t, x_{j}\right)= & x_{j}^{T}(t)\left[P_{k_{j}}\left(A_{k_{j}}+\bar{A}_{k_{j}}\right)+\left(A_{k_{j}}+\bar{A}_{k_{j}}\right)^{T} P_{k_{j}}\right] x_{j}(t) \\
- & \int_{-\tau_{k_{j}}}^{0}\left[2 x _ { j } ^ { T } ( t ) P _ { k _ { j } } \overline { A } _ { k _ { j } } \left(A_{k_{j}} x_{j}(t+\theta)\right.\right. \\
& \left.+\bar{A}_{k_{j}} x_{j}\left(t+\theta-\tau_{k_{j}}\right)\right] d \theta .
\end{aligned}
$$

Assume $V_{k_{j}}\left(t+\theta, x_{j}(t+\theta)\right)<p\left(V_{k_{j}}\left(t, x_{j}(t)\right)\right)$ for $\forall \theta \in$ $\left[-2 \tau_{k_{j}}, 0\right]$, where $p(s)=p_{k_{j}} s, p_{k_{j}}>1$, we have [3], [14]

$$
\dot{V}_{k_{j}}\left(t, x_{j}\right) \leq-x_{j}^{T}(t) S_{k_{j}}^{d} x_{j}(t),
$$

where

$$
\begin{aligned}
S_{k_{j}}^{d}:= & -\left\{P_{k_{j}}\left(A_{k_{j}}+\bar{A}_{k_{j}}\right)+\left(A_{k_{j}}+\bar{A}_{k_{j}}\right)^{T} P_{k_{j}}\right. \\
& +\tau_{k_{j}}\left[\alpha_{k_{j}}^{-1} P_{k_{j}} \bar{A}_{k_{j}} A_{k_{j}} P_{k_{j}}^{-1} \bar{A}_{k_{j}}^{T} A_{k_{j}}^{T} P_{k_{j}}\right. \\
& +\beta_{i}^{-1} P_{k_{j}}\left(\bar{A}_{k_{j}}\right)^{2} P_{k_{j}}^{-1}\left(\bar{A}_{k_{j}}^{T}\right)^{2} P_{k_{j}} \\
& \left.\left.+p_{k_{j}}\left(\alpha_{k_{j}}+\beta_{k_{j}}\right) P_{k_{j}}\right]\right\} .
\end{aligned}
$$

Because $\Sigma_{t} \in \tilde{\mathcal{A}}_{d}$, we have $S_{k_{j}}^{d}>0$ from Lemma 2.2. Therefore we can select $w(s)=w_{k_{j}}^{d} s^{2}$ in Lemma 3.1, such that (9) holds, where $w_{k_{j}}^{d}:=\sigma_{\min }\left[S_{k_{j}}^{d}\right]>0$.

Theorem 3.5: Let the dwell time be $\tau_{D}^{d}:=T_{d}^{*}+2 \tau_{\max }$, where

$$
T_{d}^{*}:=\lambda \mu_{d}\left\lfloor\frac{\lambda-1}{\bar{p}-1}+1\right\rfloor,
$$

with

$$
\mu_{d}:=\max _{i \in \mathcal{F}} \frac{\bar{\kappa}_{i}}{w_{i}^{d}}
$$

and the other parameters are the same as those defined in Theorem 3.4. Then, the system (2) with $\Sigma_{t} \in \tilde{\mathcal{A}}_{d}$ is asymptotically stable for any switching rule $q(t) \in S\left[\tau_{D}^{d}\right]$.

Proof. We can apply similar arguments used in the proof of Theorem 3.4 to obtain the following inequality:

$$
\sup _{t \in\left[t_{j}, t_{j+1}\right]}\left\|x_{j}(t)\right\| \leq \sqrt{\lambda} \delta_{j}^{d},
$$

where $\left|\psi_{j}(t)\right|_{\left[t_{j}-2 \tau_{k_{j}}, t_{j}\right]} \leq \delta_{j}^{d}$, and $\delta_{j+1}^{d}=\alpha \delta_{j}^{d}$. Note that $\delta_{0}^{d}$ can be selected as

$$
\delta_{0}^{d}:=|\psi|_{\left[-2 \tau_{\max }, 0\right]}=|\phi|_{\left[-\tau_{\max }, 0\right]}=\delta_{0} .
$$

It is clear that $|\psi|_{\left[-2 \tau_{k_{0}}, 0\right]} \leq \delta_{0}^{d}$, which further implies $\delta_{j}^{d}=$ $\delta_{j}, j \in \mathbb{Z}^{+} \cup\{0\}$. The upper bound of the state variable $x(t)$ of the switched time delay systems $\Sigma_{t}$ is bounded by a decreasing sequence $\left\{\delta_{i}\right\}, i=0,1,2, \ldots$ converging to zero, which implies the asymptotic stability and proves this theorem.

The dwell time based stability analysis proposed in this paper is general in the sense that it can be used for other stability results based on Razumikhin theorems as long as the correspondingly Lyapunov functions are in quadratic forms. Particularly, Theorem 3.5 can be extended easily to the case where $\Sigma_{t}$ has time-varying time delays and parameter uncertainties, which has important applications such as TCP (Transmission Control Protocol) congestion control of computer networks [13], [25].

\section{Conservatism Analysis}

The dwell time based stability results had been obtained for switched linear systems free of delays [10], [19]. It is interesting to compare the conservatism of the results presented in this paper with those for delay free systems.

In fact, one extreme case of the switched system $\Sigma_{t}$ is $\tau_{i}=0$ and $\bar{A}_{i}=0$ for $i \in \mathcal{A}$, which corresponds to the delay free scenario. For each candidate system $\dot{x}=A_{i} x$, a sufficient and necessary condition to guarantee asymptotic stability is $\exists P_{i}=P_{i}^{T}>0$, such that $Q_{i}:=-\left(A_{i}^{T} P_{i}+\right.$ $\left.P_{i} A_{i}\right)>0$. Correspondingly a dwell time based stability for such switched delay free system is $q(t) \in S_{\left[\tilde{\tau}_{D}\right]}$, where

$$
\tilde{\tau}_{D}=\tilde{\mu} \ln \lambda,
$$

where $\lambda$ is defined by (27) and

$$
\tilde{\mu}:=\max _{i \in \mathcal{F}} \frac{\bar{\kappa}_{i}}{\tilde{w}_{i}},
$$

where $\tilde{w}_{i}:=\sigma_{\min }\left[Q_{i}\right]>0$.

On the other hand in our case, for $\tau_{i}=0$ and $\bar{A}_{i}=0$, we observe that

$$
\lim _{\alpha_{i} \rightarrow 0^{+}} S_{i}=\lim _{\alpha_{i}, \beta_{i} \rightarrow 0^{+}} S_{i}^{d}=Q_{i}, \quad i \in \mathcal{F}
$$

from (25) and (42), which indicates $\mu=\mu_{d}=\tilde{\mu}$ by (28), (44), and (47). Accordingly we can select $p_{i}>1, i \in \mathcal{F}$ sufficiently large such that $\left\lfloor\frac{\lambda-1}{\bar{p}-1}+1\right\rfloor=1$ in (29) and (43), and obtain

$$
\tau_{D}=T^{*}=\lambda \mu=\lambda \mu_{d}=T_{d}^{*}=\tau_{D}^{d} .
$$

Therefore

$$
\tau_{D}=\tau_{D}^{d}=\lambda \tilde{\mu}>\tilde{\mu} \ln \lambda=\tilde{\tau}_{D} .
$$

The dwell times derived for switched time delay systems are proportional to $\lambda$, as opposite to the logarithm of $\lambda$ for switched delay free systems. This gap is due to the fact that asymptotic stability for linear delay free systems implies exponential stability. However, for time delay systems, the sufficient stability conditions based on LyapunovRazumikhin theorem do not guarantee exponential stability. As a matter of fact, the exponential estimates for time delay systems require additional assumptions besides asymptotic stability [15]. 
It is noticeable that stability conditions for switched time delay systems are also considered in [22], [23], where the authors give a sufficient condition to guarantee uniform stability (see Theorem 6.1 of [22] for the notation and details): $\Gamma e^{L(\Lambda+h)} \leq 1$. Apparently, this condition does not hold for the switched system (2) because in our case $\Gamma=1$, and hence

$$
\Gamma e^{L(\Lambda+h)}=e^{L(\Lambda+h)}>1, \quad \forall \Lambda>0, L>0, h>0 .
$$

The reader is referred to the journal version of this paper, [27], for numerical examples where the calculated dwell times for switched delay systems are also compared to that of delay free systems.

\section{CONCluding Remarks}

We provided stability analysis for switched linear systems with time delays, where each candidate system is assumed to be delay-independently or delay-dependently asymptotically stable. We showed the existence of a dwell time of the switching signal, such that the switched time delay system is asymptotically stable independent of the trajectory. The dwell time values for both scenarios are constructively given. The results are compared with the dwell time conditions for switched delay free systems. Optimization of the minimum dwell times we have derived, in terms of the free parameters appearing in the LMI conditions, is an interesting open problem.

\section{REFERENCES}

[1] C. Bett and M. Lemmon, "Bounded amplitude performance of switched LPV systems with applications to hybrid systems," Automatica, 35:491-503, 1999.

[2] C. De Persis, R. De Santis, and S. Morse, "Supervisory control with state-dependent dwell-time logic and constraints," Automatica, 40:269-275, 2004.

[3] L. Dugard and E.I. Verriest, Eds., Stability and Control of Time-Delay Systems, Springer, London, New York, 1998.

[4] C. Foias, H. Özbay, and A. Tannenbaum, Robust Control of Infinite Dimensional Systems: Frequency Domain Methods, Lecture Notes in Control and Information Sciences, No. 209, Springer-Verlag, London, 1996.

[5] K. Gu and S.-I. Niculescu, "Survey on recent results in the stability and control of time-delay systems," ASME Journal of Dynamic Systems, Measurement, and Control, 125:158-165, 2003.

[6] K. Gu, V.L. Kharitnov, and J. Chen, Stability and Robust Stability of Time-Delay Systems, Birkhauser, Boston, 2003.

[7] J. Hale and S. Verduyn Lunel, Introduction to Functional Differential Equations, Springer-Verlag, New York, 1993.

[8] J. Hespanha, "Uniform stability of switched linear systems: extension of Lasalle's invariance principle," IEEE Trans. Automatic Control, 49:470-482, 2004.

[9] J. Hespanha, D. Liberzon, and S. Morse, "Overcoming the limitations of adaptive control by means of logic-based switching," System \& Control Letters, 49:49-65, 2003.

[10] J. Hespanha and S. Morse, "Stability of switched systems with average dwell-time," In Proc. of the 38th Conf. on Decision and Contr., Phoenix, AZ, December 1999, pp. 2655-2660.

[11] J. Hochcerman-Frommer, S. Kulkarni, and P. Ramadge, "Controller switching based on output prediction errors," IEEE Trans. Automatic Control, 43:596-607, 1998.

[12] C. Kao and B Lincoln, "Simple stability criteria for systems with time-varying delays," Automatica, 40:1429-1434, 2004.

[13] F. Kelly, "Mathematical modelling of the Internet," in Mathematics Unlimited - 2001 and Beyond, B. Engquist and W. Schmid, Eds., Springer-Verlag, Berlin, 2001, pp. 685-702.
[14] V.L. Kharitonov, "Robust stability analysis of time delay systems: a survey," Annual Reviews in Control, 23:185-196, 1999.

[15] V.L. Kharitonov and D. Hinrichsen, "Exponential estimates for time delay systems," System \& Control Letters, 53(5):395-405, 2004.

[16] V. Kulkarni, M. Jun, and J. Hespanha, "Piecewise quadratic Lyapunov functions for piecewise affine time delay systems," in Proc. of the American Control Conference, Boston, MA, June-July 2004, pp. 38853889.

[17] D. Liberzon and S. Morse, "Basic problems in stability and design of switched systems," IEEE Control Systems Magazine, 19:59-70, 1999.

[18] V. Montagner, V. Leite, S. Tarbouriech, and P. Peres, "Stability and stabilizability of discrete-time switched linear systems with state delay," in Proc. of the American Control Conference, 2005, Portland OR, June 2005, pp. 3806-3811.

[19] S. Morse, "Supervisory control of families of linear set-point controllers: part 1: exact matching," IEEE Trans. Automatic Control, 41:1413-1431, 1996.

[20] S.-I. Niculescu, Delay Effects on Stability: A Robust Control Approach, Lecture Notes in Control and Information Sciences No. 269, SpringerVerlag, Heidelberg, 2001.

[21] E. Skafidas, R. Evans, A. Savkin, and I. Peterson, "Stability results for switched controller systems," Automatica, 35:553-564, 1999.

[22] Y. Sun, A Michel, and G. Zhai, "Stability of discontinuous retarded functional differential equations with applications to delay systems," in Proc. of the American Control Conference, Denver, CO, June 2003, pp. 3387-3392.

[23] Y. Sun, A Michel, and G. Zhai, "Stability of discontinuous retarded functional differential equations with applications," IEEE Trans. Automatic Control, 50:1090-1105, 2005.

[24] F. Wu and K. Grigoriadis, "LPV systems with parameter-varying time delays: analysis and control," Automatica, 37:221-229, 2001.

[25] P. Yan and H. Özbay, "Robust controller design for AQM and $\mathcal{H}^{\infty}$ performance analysis," in Advances in Communication Control Networks, S. Tarbouriech, C. Abdallah, and J. Chiasson, Eds., Lecture Notes in Control and Information Sciences, No. 308, Springer-Verlag, New York, 2005, pp. 49-64.

[26] P. Yan and H. Özbay, "On switching $\mathcal{H}^{\infty}$ controllers for a class of linear parameter varying systems," System \& Control Letters, 56(78):504-511, 2007.

[27] P. Yan and H. Özbay, "Stability Analysis of switched time-delay systems," SIAM Journal on Control and Optimizations, 47(2):936949, 2008.

[28] D. Yue and Q Han, "Delay-dependent exponential stability of stochastic systems with time-varying delay, nonlinearity, and Markovian switching," IEEE Trans. Automatic Control, 50:217-222, 2005.

[29] G. Zhai, Y. Sun, X. Chen, and A. Michel, "Stability and $\mathcal{L}^{2}$ gain analysis for switched symmetric systems with time delay," in Proc. of the American Control Conference, Denver, CO, June 2003, pp. 26822687. 